\title{
Ultrasound-targeted Microbubble Destruction Promotes Myocardial Angiogenesis and Functional Improventments in Rat Model of Diabetic Cardiomyopathy
}

\author{
Xijun Zhang \\ zhengzhou University People's Hospital \\ Haohui Zhu \\ Zhengzhou Uninversity People's Hospital \\ Nanqian Zhou \\ Zhengzhou University People's Hospital \\ Zhixin Fang \\ Xinxiang Medical University \\ Yuping Yang \\ Xinxiang Medical University \\ Yun Jing \\ Xinxiang Medical University \\ Jianjun Yuan (D 342354073@qq.com ) \\ zhengzhou university people's Hospital \\ Peng Li \\ Xinxiang Medical University \\ Xinqiao Tian \\ Zhengzhou University People's Hospital
}

Original investigation

Keywords: Diabetic cardiomyopathy, Ultrasound microbubble contrast, Angiogenesis, Animal model

Posted Date: August 14th, 2020

DOI: https://doi.org/10.21203/rs.3.rs-33215/v2

License: () (1) This work is licensed under a Creative Commons Attribution 4.0 International License.

Read Full License 
The authors have withdrawn this preprint from Research Square 